\title{
Lessons learnt from hydroxychloroquine/azithromycin in treatment of COVID-19
}

\author{
Jiuyang Xu $\mathbb{1}^{1,2,3}$ and Bin $\mathrm{Cao}^{2,3,4}$
}

${ }^{1}$ Tsinghua University School of Medicine, Beijing, China. ${ }^{2}$ Dept of Pulmonary and Critical Care Medicine, Center of Respiratory Medicine, China-Japan Friendship Hospital, National Center for Respiratory Medicine, National Clinical Research Center for Respiratory Diseases, Beijing, China. ${ }^{3}$ Institute of Respiratory Medicine, Chinese Academy of Medical Sciences, Beijing, China. ${ }^{4}$ Tsinghua UniversityPeking University Joint Center for Life Sciences, Beijing, China.

Corresponding author: Bin Cao (caobin_ben@163.com)

Shareable abstract (@ERSpublications)

Solid pre-clinical investigations serve as the basis for clinical research. High quality clinical trials remain the definitive measure to evaluate an interventional therapy. Clinical decisions should be independent from media and political propaganda. https://bit.ly/3iaNBsB

Cite this article as: Xu J, Cao B. Lessons learnt from hydroxychloroquine/azithromycin in treatment of COVID-19. Eur Respir J 2022; 59: 2102002 [DOI: 10.1183/13993003.02002-2021].
Copyright (C)The authors 2022.

This version is distributed under the terms of the Creative Commons Attribution NonCommercial Licence 4.0. For commercial reproduction rights and permissions contact permissions@ersnet.org

Received: 18 July 2021 Accepted: 19 July 2021

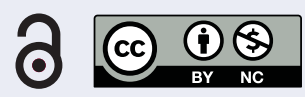

Coronavirus disease 2019 (COVID-19) is still exerting a great influence on global health and economy. The initial fight against COVID-19 relied mainly on non-pharmacological measures, including social distancing, facial masks, active screening and isolation, etc. In terms of pharmacological interventions, huge progress has been made in the development of vaccines against severe acute respiratory syndrome coronavirus 2 (SARS-CoV-2), although the appearance of several mutant variants has raised concerns for lasting immunity. The repurposing of established drugs has been another strategy to search for weapons in treatment or prophylaxis against COVID-19. Although immuno-modulating drugs, including corticosteroids, have been demonstrated to have therapeutic effects, no anti-viral medications have so far been shown to be protective.

Initially developed as an anti-malarial drug, chloroquine/hydroxychloroquine has later been widely used in treatment of rheumatic diseases, including systemic lupus erythematosus and rheumatoid arthritis [1]. Some potential anti-viral effect of chloroquine has previously been reported (including in SARS-CoV and human coronavirus OC43 infection) [2-6] but the evidence was mainly pre-clinical and the results were mixed. Chloroquine/hydroxychloroquine has been the "darling" of the scientific community since the beginning of the COVID-19 outbreak. It was reported early in the pandemic that chloroquine had anti-viral effects against SARS-CoV-2 in an in vitro cell line model [7]. Later, its less toxic derivative hydroxychloroquine was demonstrated to share similar, yet slightly less potent, effects in cell models [8]. A preliminary retrospective analysis reported faster viral clearance in COVID-19 patients treated with hydroxychloroquine (and with combination of hydroxychloroquine and azithromycin) compared with standard care [9], but the study design and data interpretation remained controversial.

Despite lack of further evidence, clinical trials of hydroxychloroquine to treat COVID-19 were soon launched around the globe. Upon writing of the manuscript, there are already 281 registered trials (on clinicaltrials.gov) on hydroxychloroquine treatment for COVID-19, and some are still recruiting. Hydroxychloroquine was even praised as a "game-changer" against coronavirus by political figures and was soon granted emergency use authorisation.

Although some researchers promoted the use of hydroxychloroquine (as well as its combination with azithromycin) [10], others reported neutral results in observational studies [11, 12] and small-scale interventional trials [13]. No protective effects were found in post-exposure prophylaxis studies either [14]. Further, the risk of adverse effects of hydroxychloroquine, including elongation of QT intervals, has raised concern $[15,16]$. In this rush to find a cure for COVID-19, the community awaits high-quality randomised clinical trials to draw final conclusions. 
The ProPAC-COVID study group conducted a double-blinded placebo-controlled trial to study the effect of hydroxychloroquine and azithromycin in treatment of hospitalised PCR-confirmed COVID-19 patients [17]. The intervention group was administered a moderate dose of hydroxychloroquine and azithromycin for 15 days, whereas the control group received placebo/placebo and standard care. The trial was pre-terminated due to futility at a pre-planned interim analysis. Researchers concluded that the combination of azithromycin and hydroxychloroquine did not improve survival or length of hospitalisation in COVID-19 patients. This is in line with the latest recommendation from the European Respiratory Society guidelines [18].

Previously, the open-label RECOVERY and SOLIDARITY trials have reported neutral effects for hydroxychloroquine and azithromycin in hospitalised COVID-19 patients [19-21]. The Coalition I trial from Brazil further demonstrated that the combination of these two drugs did not improve clinical status in mild-to-moderate COVID-19 patients [22]. Although the ProPAC-COVID trial is not the first or largest trial so far to study the effect of hydroxychloroquine against COVID-19, its strength lies in the double-blinded and placebo-controlled design, when outcomes such as ordinal scales and hospital discharge may be influenced by physicians. Another strength is that all trial participants had been laboratory-confirmed by PCR. The pre-termination of the trial at interim analysis and relatively small sample size was the major limitation, but in general this well-designed and well-adhered trial provides valuable information for the discussion of hydroxychloroquine in COVID-19. It also emphasises the critical role of high-quality randomised controlled trials in evidence-based medicine, especially during such a serious pandemic.

Hydroxychloroquine and azithromycin were among the most commonly used repurposed drugs in the COVID-19 pandemic. Triggered by optimistic pre-clinical and early clinical reports, and perhaps also by the fear of the unknown disease and eagerness of finding a cure, the use of hydroxychloroquine increased rapidly from March to April, 2020 [23]. Later, its use steeply decreased in May and June, 2020, as more clinical evidence was gradually released. The subsequent pre-clinical investigations also revealed that the anti-viral effect of hydroxychloroquine in monkey kidney cell lines (VeroE6, as reported earlier in [8]) does not translate into inhibition of viral replication in primary respiratory epithelium cells. Nor does it confer protection against SARS-CoV-2 infection in primate animal models [24, 25]. The discrepancy between in vivo and in vitro phenotype should have halted the push of hydroxychloroquine to human use for treatment of COVID-19, but it was too late, as many clinical trials had already been launched and emergency use been authorised.

How it is possible to cope with research and the search for a treatment against a newly emerged pathogen, especially during a pandemic setting? There is a lot to learn from the story of hydroxychloroquine in COVID-19. First, solid pre-clinical investigations always serve as the basis for clinical research. The process from compound screening, in vitro assays and confirmation in animal models, all the way to clinical trials, can be accelerated in such pandemic times, but none of the pre-clinical procedures should be skipped in order to save time. In fact, the so-called timely breakthroughs in "war time" rely heavily on the numerous preparations finished during "peace time”. Investment into scientific research cannot be halted should the pandemic cease. Secondly, high quality clinical trials, whenever practical and possible, remain the definitive measure for evaluating an interventional therapy. Retrospective analysis and observational studies may provide timely and useful information, but care must be taken when evaluating such evidence and cross validation from multiple cohorts/studies is optimal. Conclusions drawn from a few or a single cohort, especially when the sample number is limited, may be biased. High level evidence from well-designed, strictly adhered to, and preferably large-scale randomised clinical trials is important for making clinical decisions. The experience from COVID-19 clinical research demonstrates that rapidly conducting high-level randomised controlled trials is feasible during such a pandemic, and that simple, large, platform trials may be most powerful. Thirdly, clinical decisions should be independent from media and political propaganda. Keeping calm and objective can be challenging in the rush to find a cure, but it is necessary to remain neutral. Furthermore, the pandemic is putting forward higher expectations for physicians and scientists: correct information has to be conveyed directly and precisely to the general public. We need to get prepared.

Facing a new disease, decisions are made from the best available evidence, and will be further modified when new evidence emerges. Some choices that are deemed wrong or inappropriate might have been "correct" provided with the limited evidence at an earlier time. In this process, experience is accumulated, medicine is advanced, and empirical medicine turns into evidence-based medicine.

We have learnt a lot from the current COVID-19 pandemic. Are we prepared for the next challenge?

Conflict of interest: J. Xu has nothing to disclose. B. Cao has nothing to disclose. 
References

$1 \quad$ Ben-Zvi I, Kivity S, Langevitz P, et al. Hydroxychloroquine: from malaria to autoimmunity. Clin Rev Allergy Immunol 2012; 42: 145-153.

2 Barnard DL, Day CW, Bailey $\mathrm{K}$, et al., Evaluation of immunomodulators, interferons and known in vitro SARS-CoV inhibitors for inhibition of SARS-CoV replication in BALB/c mice. Antivir Chem Chemother 2006; 17: 275-284.

3 Keyaerts E, Li S, Vijgen L, et al. Antiviral activity of chloroquine against human coronavirus OC43 infection in newborn mice. Antimicrob Agents Chemother 2009; 53: 3416-3421.

4 Keyaerts E, Vijgen L, Maes $\mathrm{P}$, et al. In vitro inhibition of severe acute respiratory syndrome coronavirus by chloroquine. Biochem Biophys Res Commun 2004; 323: 264-268.

5 Niu J, Shen L, Huang B, et al. Non-invasive bioluminescence imaging of HCoV-OC43 infection and therapy in the central nervous system of live mice. Antiviral Res 2020; 173: 104646.

6 Vincent MJ, Bergeron E, Benjannet S, et al. Chloroquine is a potent inhibitor of SARS coronavirus infection and spread. Virol J 2005; 2: 69.

7 Wang $\mathrm{M}$, Cao R, Zhang L, et al. Remdesivir and chloroquine effectively inhibit the recently emerged novel coronavirus (2019-nCoV) in vitro. Cell Research 2020; 30: 269-271.

8 Liu J, Cao R, Xu M, et al. Hydroxychloroquine, a less toxic derivative of chloroquine, is effective in inhibiting SARS-CoV-2 infection in vitro. Cell Discovery 2020; 6: 16.

9 Gautret P, Lagier JC, Parola P, et al. Hydroxychloroquine and azithromycin as a treatment of COVID-19: results of an open-label non-randomized clinical trial. Int J Antimicrob Agents 2020; 56: 105949.

10 Arshad S, Kilgore P, Chaudhry ZS, et al. Treatment with hydroxychloroquine, azithromycin, and combination in patients hospitalized with COVID-19. Int J Infect Dis 2020; 97: 396-403.

11 Rosenberg ES, Dufort EM, Udo T, et al. Association of treatment with hydroxychloroquine or azithromycin with in-hospital mortality in patients with COVID-19 in New York State. JAMA 2020; 323: 2493-2502.

12 Geleris J, Sun Y, Platt J, et al. Observational study of hydroxychloroquine in hospitalized patients with Covid-19. N Engl J Med 2020; 382: 2411-2418.

13 Tang W, Cao Z, Han M, et al. Hydroxychloroquine in patients with mainly mild to moderate coronavirus disease 2019: open label, randomised controlled trial. BMJ 2020; 369: m1849.

14 Boulware DR, Pullen MF, Bangdiwala AS, et al. A randomized trial of hydroxychloroquine as postexposure prophylaxis for Covid-19. N Engl J Med 2020; 383: 517-525.

15 Borba MGS, Val FFA, Sampaio VS, et al. Effect of high vs low doses of chloroquine diphosphate as adjunctive therapy for patients hospitalized with severe acute respiratory syndrome coronavirus 2 (SARS-CoV-2) infection: a randomized clinical trial. JAMA Netw Open 2020; 3: e208857.

16 Chorin E, Dai M, Shulman E, et al. The QT interval in patients with COVID-19 treated with hydroxychloroquine and azithromycin. Nat Med 2020; 26: 808-809.

17 Sivapalan P, Ulrik CS, Lapperre TS, et al. Azithromycin and hydroxychloroquine in hospitalised patients with confirmed COVID-19: a randomised double-blinded placebo-controlled trial. Eur Respir J 2022; 59: 2100752.

18 Chalmers JD, Crichton ML, Goeminne PC, et al. Management of hospitalised adults with coronavirus disease 2019 (COVID-19): a European Respiratory Society living guideline. Eur Respir J 2021; 57: 2100048.

19 Abaleke E, Abbas M, Abbasi S, et al. Azithromycin in patients admitted to hospital with COVID-19 (RECOVERY): a randomised, controlled, open-label, platform trial. Lancet 2021; 397: 605-612.

20 Pan H, Peto R, Henao-Restrepo AM, et al. Repurposed antiviral drugs for Covid-19 - interim WHO Solidarity Trial Results. N Engl J Med 2021; 384: 497-511.

21 Horby P, Mafham M, Linsell L, et al. Effect of hydroxychloroquine in hospitalized patients with Covid-19. N Engl J Med 2020; 383: 2030-2040.

22 Cavalcanti $A B$, Zampieri FG, Rosa RG, et al. Hydroxychloroquine with or without azithromycin in mild-to-moderate Covid-19. N Engl J Med 2020; 383: 2041-2052.

23 Prats-Uribe A, Sena AG, Lai LYH, et al. Use of repurposed and adjuvant drugs in hospital patients with Covid-19: multinational network cohort study. BMJ 2021; 373: n1038.

24 Hoffmann M, Mösbauer K, Hofmann-Winkler H, et al. Chloroquine does not inhibit infection of human lung cells with SARS-CoV-2. Nature 2020; 585: 588-590.

25 Maisonnasse P, Guedj J, Contreras V, et al. Hydroxychloroquine use against SARS-CoV-2 infection in non-human primates. Nature 2020; 585: 584-587. 